Pesquisa Florestal Brasileira
Brazilian Journal of Forestry Research
http://pfb.cnpf.embrapa.br/pfb/ $\quad \begin{aligned} & \text { ISSN: 1983-2605 (online) } \\ & \text { Nota Científica }\end{aligned}$

\title{
Propriedades físico-mecânicas da madeira de uva-do-japão
}

\author{
Éverton Hillig ${ }^{1 *(D)}$, Tiago Digner $^{2}$ (D) Andrea Nogueira Dias ${ }^{1}$ (D) \\ ${ }^{1}$ Universidade Estadual do Centro-Oeste, Departamento de Engenharia Florestal, BR 153, Km 07, CEP 84500-000, Irati, PR, Brasil \\ ${ }^{2}$ Duratex Madeiras Agudos, Rod. Mal. Rondon, s/n, Zona Rural, CEP 17120-000, Agudos, SP, Brasil
}

\author{
"Autor correspondente: \\ hillig@hotmail.com \\ Termos para indexação: \\ Massa específica \\ Resistência mecânica \\ Hovenia dulcis \\ Index terms: \\ Specific gravity \\ Mechanical strength \\ Hovenia dulcis \\ Histórico do artigo: \\ Recebido em 21/03/16 \\ Aprovado em 23/05/18 \\ Publicado em 10/10/18 \\ doi: 10.4336/2018.pfb.38e201601195
}

Resumo - Foram avaliadas as propriedades físico-mecânicas da madeira de Hovenia dulcis Thumberg, proveniente de seis árvores e retiradas de três posições no fuste. A massa específica aparente média foi de $0,64 \mathrm{~g} \mathrm{~cm}^{-3}$ e a umidade de equilíbrio, após condicionamento a $20{ }^{\circ} \mathrm{C}$ e $65 \%$ de umidade relativa do ar, foi de $15,5 \%$. Houve diferença das propriedades físicas da madeira entre árvores e posição no fuste. Os valores médios das propriedades mecânicas indicam enquadramento na classe C20 de classificação para madeiras de dicotiledôneas da Norma NBR 7190/97, exceto para módulo de elasticidade em compressão paralela.

\section{Physical and mechanical wood properties of Japanese raisin tree}

\begin{abstract}
We evaluated physical and mechanical wood properties of Hovenia dulcis Thumberg, from six trees. Samples were taken from three different positions in the stem. The mean apparent specific gravity was $0.64 \mathrm{~g} \mathrm{~cm}^{-3}$ and the moisture content after conditioning at $20^{\circ} \mathrm{C}$ and $65 \%$ relative humidity was $15.5 \%$. Physical wood properties were different among trees and stem position. The average mechanical properties values indicate a C20 timber class when applying NBR 7190/97 hardwood classification, except for the modulus of elasticity in parallel compression.
\end{abstract}

Hovenia dulcis Thunberg é uma espécie nativa da China, Coréia do Norte, Coréia do Sul e Japão, da família Rhamnaceae, sendo popularmente conhecida como uvado-japão. É uma árvore caducifólia, que normalmente atinge $10 \mathrm{~m}$ a $15 \mathrm{~m}$ de altura e $20 \mathrm{~cm}$ a $40 \mathrm{~cm}$ de diâmetro a $1,30 \mathrm{~m}$ do solo (DAP). O seu tronco normalmente é reto e cilíndrico, podendo apresentar fuste com até $8 \mathrm{~m}$ de comprimento, ramificação dicotômica, copa globosa e ampla. Apresenta gemas dormentes, podendo ser manejada por talhadia (Rigatto et al., 2001). No Paraná, essa espécie encontra-se em alguns pequenos plantios experimentais e associadas às florestas nativas remanescentes, pois se trata de uma espécie de fácil regeneração (Carvalho, 1994).

É uma espécie que apresenta crescimento rápido, mostrando grande potencial para produção de matériaprima de madeira serrada. Schumacher et al. (2008) avaliaram a produção de biomassa de um plantio de $H$. dulcis aos 18 anos de idade, na região de Santa Maria, RS, e observaram que a biomassa estimada alcançou 181,6 $\mathrm{Mg} \mathrm{ha}^{-1}$, sendo $68,6 \%$ de madeira, $15,5 \%$ de galhos (vivos e mortos), $11,2 \%$ de cascas e $4,7 \%$ de 
folhas. Eleotério et al. (2012) avaliaram o crescimento em diâmetro de árvores da mesma espécie na região de Blumenau, SC, verificando que o valor máximo obtido para incremento médio anual (IMA) e para o incremento corrente anual (ICA) foi de 1,29 cm e $1,43 \mathrm{~cm}$, respectivamente, em árvores com 20 anos.

De acordo com Rigatto et al. (2001), a madeira de $H$. dulcis é moderadamente pesada. Seu tronco apresenta alburno amarelo e cerne castanho-escuro ou vermelho, com brilho opaco a mediano. É uma madeira que não tem cheiro, com textura fina homogênea e grã direita, sendo fácil de trabalhar com ferramentas, resultando em superfícies lisas e brilhantes. É considerada madeira de boa resistência, medianamente tenaz e elástica, mas pouco durável em contato com o solo e susceptível ao ataque de fungos (Carvalho et al., 2015; Tomazeli et al., 2015).

Susin et al. (2014) avaliaram a qualidade da madeira de $H$. dulcis submetida à secagem ao ar e em estufa solar, sendo que a madeira não apresentou, em quaisquer dos métodos, redução na qualidade em função da ocorrência de defeitos. Segundo Vivian et al. (2010), a indústria madeireira e moveleira da região de Caxias do Sul, RS contava com plantios dessa espécie em pequena escala com bons resultados. No entanto, são poucas as informações sobre as formas adequadas de beneficiamento da madeira, o que é indispensável para seu uso na indústria moveleira.

Dessa forma, este trabalho teve como objetivo avaliar as propriedades físico-mecânicas da madeira de $H$. dulcis, verificando a variação que ocorre entre árvores e ao longo do fuste. Foi também determinada a correlação entre as propriedades mecânicas estudadas e entre essas e a massa específica da madeira.

Foram colhidas, aleatoriamente, seis árvores de $H$. dulcis em remanescentes de floresta natural no Município de Irati, PR ( $25^{\circ} 27^{\prime} 56^{\prime}$ 'S e $\left.50^{\circ} 37^{\prime} 51^{\prime \prime} \mathrm{W}\right)$. A região está situada a uma altitude de aproximadamente $812 \mathrm{~m}$, apresentando clima tipo $\mathrm{Cfb}$ (temperado) de acordo com a classificação climática de Köppen, com verões amenos, invernos com ocorrências de geadas severas e frequentes, sem estação seca (Instituto Agronômico do Paraná, 2015).

As árvores foram desdobradas em três toras de $3 \mathrm{~m}$ cada, marcadas sequencialmente (T1, T2 e T3). A árvore quatro teve a terceira tora de menor comprimento, em função de sua altura comercial menor.

De cada tora, foram desdobradas vigas de $6 \mathrm{~cm} \times 6 \mathrm{~cm}$ $\mathrm{x} 100 \mathrm{~cm}$, que foram submetidas a secagem ao ar, por aproximadamente dois meses. Após, foram selecionadas as vigas sem defeitos para a confecção dos corpos-deprova.

Os corpos-de-prova confeccionados foram submetidos à climatização em ambiente com $65 \%$ de umidade relativa do ar e $20^{\circ} \mathrm{C}$. Os ensaios físicos para determinação do teor de umidade e massa específica aparente da madeira foram realizados utilizando os corpos de prova dos ensaios mecânicos (Comisión Panamericana de Normas Técnicas, 1972a, 1972b). Os ensaios mecânicos de cisalhamento, compressão paralela às fibras, dureza (no sentido paralelo e perpendicular às fibras) e flexão estática foram realizados em Máquina Universal de Ensaios EMIC - DL 30.000 (Comisión Panamericana de Normas Técnicas, 1972c, 1972d, 1972e, 1973).

Os valores médios do teor de umidade de equilíbrio (TUe) e da massa específica aparente (MEa) de cada árvore e tora (posição da madeira no fuste), juntamente com os valores dendrométricos de cada árvore (DAP e altura comercial - Hc) estão apresentados na Tabela 1.

Tabela 1. Valores médios de teor de umidade de equilíbrio e de massa específica aparente por árvore e por tora (posição na árvore) e variáveis dendrométricas de árvores de Hovenia dulcis provenientes de remanescentes de floresta natural no município de Irati, PR.

Table 1. Mean values of equilibrium moisture content and apparent density per tree and log (tree position) and dendrometric variables of Hovenia dulcis trees from natural forest remnants in the municipality of Irati, PR.

\begin{tabular}{cccccc}
\hline Fator & Nível & $\begin{array}{c}\text { DAP } \\
(\mathbf{c m})\end{array}$ & $\begin{array}{c}\text { Hc } \\
\mathbf{( m )}\end{array}$ & $\begin{array}{c}\text { TUe } \\
\mathbf{( \% )}\end{array}$ & $\begin{array}{c}\text { MEa } \\
\left(\mathbf{g ~ c m}^{-3}\right)\end{array}$ \\
\hline & 1 & 41,7 & 11,5 & $16,0 \mathrm{a}$ & $0,67 \mathrm{a}$ \\
Árvore & 2 & 26,1 & 12,0 & $16,0 \mathrm{a}$ & $0,64 \mathrm{bc}$ \\
& 3 & 25,8 & 12,0 & $15,2 \mathrm{bc}$ & $0,60 \mathrm{~d}$ \\
& 4 & 23,9 & 8,8 & $15,5 \mathrm{ab}$ & $0,61 \mathrm{~cd}$ \\
& 5 & 29,6 & 13,5 & $15,4 \mathrm{ab}$ & $0,67 \mathrm{ab}$ \\
\hline Fcalculado & 6 & 22,6 & 11,5 & $14,8 \mathrm{c}$ & $0,63 \mathrm{~b}$ \\
\hline & 1 & --- & --- & $16,1 \mathrm{a}$ & $0,65 \mathrm{a}$ \\
Tora & 2 & --- & --- & $15,2 \mathrm{~b}$ & $0,62 \mathrm{~b}$ \\
& 3 & --- & --- & $15,0 \mathrm{~b}$ & $0,65 \mathrm{a}$ \\
\hline Fcalculado & & & & $30,9 *$ & $12,28^{*}$ \\
\hline Interação & & & & $7,9 *$ & $2,28^{*}$ \\
\hline Média geral & & & & 15,5 & 0,64 \\
\hline CV (\%) & & & & 5,8 & 4,7 \\
\hline
\end{tabular}

$\overline{\mathrm{DAP}}=$ diâmetro a $1,30 \mathrm{~m}$ do solo; $\mathrm{Hc}=$ altura comercial, $\mathrm{TUe}=$ teor $\mathrm{de}$ umidade de equilíbrio; $\mathrm{MEa}=$ massa específica aparente; $\mathrm{CV}=$ coeficiente de variação; *significativo a $5 \%$ de probabilidade de erro. Médias seguidas da mesma letra, na coluna, não diferem entre si pelo teste de Tukey, ao nível de $5 \%$ de probabilidade de erro. 
O valor médio de massa específica aparente (MEa), de $0,64 \mathrm{~g} \mathrm{~cm}^{-3}$ está de acordo com os valores encontrados por Eleotério et al. (2015), Napoli et al. (2013) e Rigato et al. (2001), de $0,54 \mathrm{~g} \mathrm{~cm}^{-3}, 0,57 \mathrm{~g} \mathrm{~cm}^{-3} \mathrm{e}$ de $0,50 \mathrm{~g} \mathrm{~cm}^{-3}$ a $0,72 \mathrm{~g} \mathrm{~cm}^{-3}$, para massa específica básica, respectivamente.

$\mathrm{O}$ teor de umidade de equilíbrio médio (TUe) foi de $15,5 \%$ que, embora alto, é semelhante ao valor encontrado por Susin et al. (2014), de 15,4\% para madeira de $H$. dulcis submetida ao processo de secagem ao ar com temperatura média diária de $18,0{ }^{\circ} \mathrm{C}$ e média de umidade relativa do ar de $60 \%$.

Houve diferença de TUe entre árvores e entre toras. Mendes \& Arce (2003) apresentaram valores estimados de TUe de madeira para o estado do Paraná variando de 12,87 a 16,09. Foi utilizado o método por estufa para determinação do TUe da madeira de Hovenia dulcis utilizada neste estudo, que pode incluir alguns extrativos além da água, e Rigatto et al. (2001) encontraram para a madeira da espécie teor de extrativos de 7\%. Assim, o maior TUe da tora 1 pode ser atribuído ao maior teor de extrativos desta tora, em função da maior proporção de cerne.

Observou-se diferença de MEa entre árvores e entre toras, além de ocorrer interação entre esses fatores (Tabela 1), não sendo possível estabelecer um padrão de variação da $\mathrm{MEa}$ em função da posição (altura) no fuste, corroborando resultados observados na literatura. Candaten et al. (2017) verificaram variação de massa específica básica (MEb) da madeira de $H$. dulcis entre árvores $\left(0,436 \mathrm{~g} \mathrm{~cm}^{-3}\right.$ a $\left.0,619 \mathrm{~g} \mathrm{~cm}^{-3}\right)$ e diminuição dessa propriedade com a altura no fuste, sendo essa tendência mais pronunciada a partir de $50 \%$ da altura. No entanto, Eleotério et al. (2015) verificaram aumento da MEb com o aumento da altura no fuste em árvores de $\mathrm{H}$. dulcis com DAP médio de $23,7 \mathrm{~cm}$ e altura total média de $22,0 \mathrm{~m}$.

Em relação às variáveis dendrométricas, verificou-se maior massa específica da madeira (ME) das árvores de maior DAP, que eram as mais velhas, ou aquelas com maior taxa de crescimento. É de conhecimento explícito e do senso comum que a ME aumenta com a idade da árvore, no entanto, essa característica pode variar. Trevisan et al. (2016) não encontraram um padrão de variação para ME da madeira de araucária proveniente de árvores de diferentes classes diamétricas. Para folhosas, a influência da taxa de crescimento na massa específica da madeira pode ser mais variável do que para coníferas, pois a estrutura anatômica mais complexa e as menores diferenças entre madeira juvenil e adulta provocam maiores variações na espessura da parede celular que, por sua vez, determina a massa específica da madeira. Os lenhos juvenis e adultos são influenciados pelas condições de crescimento, tratos silviculturais e origem genética e, assim, refletem nas propriedades da madeira (Vidaurre et al., 2011).

Na Tabela 2 estão apresentados os valores médios das propriedades mecânicas avaliadas, por árvore e por tora. Exceto para o módulo de elasticidade em compressão (EC) e para dureza no sentido transversal às fibras, observou-se diferença entre as árvores. Em relação à posição no fuste, somente o módulo de ruptura em flexão estática não apresentou diferença. Sendo a madeira um material natural e estando as árvores sujeitas a diferentes condições de umidade, solos e fase de crescimento, as suas propriedades mecânicas variam consideravelmente (Forest Products Laboratory, 2010), resultando em diferenças entre árvores e entre toras. Por outro lado, verificou-se que o coeficiente de variação calculado para as propriedades mecânicas é semelhante ou menor ao observado por Müller et al. (2014) para madeira de eucalipto.

Na Tabela 3 é apresentada a correlação de Pearson entre as propriedades físico-mecânicas estudadas. Verificou-se que o TUe só se correlacionou com a MEa e com a resistência ao cisalhamento. A $\mathrm{MEa}$, por sua vez, apresentou correlação significativa com todas as outras propriedades estudadas. Todas as propriedades mecânicas tiveram seus valores aumentados com o aumento da massa específica aparente. Autores que avaliaram as propriedades físicas e mecânicas de algumas espécies folhosas verificaram a relação positiva da massa específica com as propriedades de resistência e rigidez da madeira (Araújo, 2007; Del Menezzi et al., 2010; Tenorio et al., 2016), enquanto Talgatti et al. (2017) verificaram boa relação $\left(r^{2}=0,81\right)$ entre a massa específica aparente e a flexão dinâmica da madeira de H. dulcis.

As propriedades mecânicas em função da massa específica aparente, juntamente com a equação de regressão linear, para as propriedades que apresentaram coeficiente de determinação maior que 0,5 estão apresentadas na Figura 1. Dias \& Lahr (2004) verificaram coeficientes de 0,76 (MOR), 0,71 (MOE), 0,92 (DurA) e 0,71 (DurT) que foram superiores aos encontrados nesse estudo, mas que foram obtidos a partir da avaliação de 40 espécies florestais o que proporcionou maior variabilidade dos dados. 
Tabela 2. Valores médios das propriedades mecânicas por árvore e por tora (posição no fuste) da madeira de árvores de Hovenia dulcis provenientes de remanescentes de floresta natural no Município de Irati, PR.

Table 2. Mean values of mechanical properties per tree and log (tree position) of Hovenia dulcis wood from natural forest remnants in the Municipality of Irati, PR.

\begin{tabular}{|c|c|c|c|c|c|c|c|c|}
\hline Fator & Nível & $\begin{array}{l}\text { MOR } \\
\text { (MPa) }\end{array}$ & $\begin{array}{l}\text { MOE } \\
\text { (MPa) }\end{array}$ & $\begin{array}{c}\mathrm{RC} \\
(\mathrm{MPa}) \\
\end{array}$ & $\begin{array}{c}\text { EC } \\
(\mathrm{MPa})\end{array}$ & $\begin{array}{l}\text { DurA } \\
\text { (KN) }\end{array}$ & $\begin{array}{l}\text { DurT } \\
\text { (KN) }\end{array}$ & $\begin{array}{c}\text { Cis } \\
(\mathrm{MPa})\end{array}$ \\
\hline \multirow{6}{*}{ Árvore } & 1 & $98,4 a$ & $10.993 \mathrm{ab}$ & $43,4 a$ & $4.606 a$ & $5,26 \mathrm{ab}$ & $4,82 \mathrm{a}$ & $15,2 \mathrm{~b}$ \\
\hline & 2 & $89,7 \mathrm{a}$ & $11.076 \mathrm{ab}$ & $36,4 \mathrm{bc}$ & $4.307 \mathrm{a}$ & $5,06 \mathrm{ab}$ & $4,69 a$ & $15,3 b$ \\
\hline & 3 & $70,8 \mathrm{bc}$ & $9.456 \mathrm{~b}$ & $36,4 \mathrm{bc}$ & $4.001 \mathrm{a}$ & $4,34 \mathrm{ab}$ & $4,38 \mathrm{a}$ & $13,1 \mathrm{c}$ \\
\hline & 4 & $52,2 \mathrm{c}$ & $6.171 \mathrm{c}$ & $34,6 \mathrm{c}$ & $3.588 \mathrm{a}$ & $4,15 b$ & $4,07 \mathrm{a}$ & $13,1 \mathrm{c}$ \\
\hline & 5 & $98,0 \mathrm{a}$ & $13.464 \mathrm{a}$ & $41,2 \mathrm{ab}$ & $4.403 \mathrm{a}$ & $5,47^{\mathrm{a}}$ & $4,94 \mathrm{a}$ & $16,1 b$ \\
\hline & 6 & $80,0 \mathrm{ab}$ & $10.229 b$ & $40,9 \mathrm{ab}$ & $4.212 \mathrm{a}$ & $4,52 \mathrm{ab}$ & $4,42 \mathrm{a}$ & $17,9 \mathrm{a}$ \\
\hline Fcalculado & & $10,5^{*}$ & $13,5^{*}$ & $8,7^{*}$ & $2,34^{\mathrm{ns}}$ & $3,7 *$ & $2,1^{\text {ns }}$ & $24,1 *$ \\
\hline \multirow{3}{*}{ Tora } & 1 & $77,7 \mathrm{a}$ & $9.623 b$ & $38,2 b$ & $4.224 \mathrm{ab}$ & $4,89 \mathrm{ab}$ & $4,60 \mathrm{ab}$ & $14,6 b$ \\
\hline & 2 & $79,7 \mathrm{a}$ & $9.979 b$ & $36,5 b$ & $3.823 b$ & $4,62 b$ & $4,27 b$ & $14,8 \mathrm{~b}$ \\
\hline & 3 & $89,6 \mathrm{a}$ & $12.141 \mathrm{a}$ & $42,5 \mathrm{a}$ & $4.607 \mathrm{a}$ & $5,26 \mathrm{a}$ & $5,03 \mathrm{a}$ & $16,3 \mathrm{a}$ \\
\hline Fcalculado & & $0,9^{\text {ns }}$ & $5,8^{*}$ & $10,4^{*}$ & $7,6^{*}$ & $1,3^{\mathrm{ns}}$ & $6,9^{*}$ & $8,3^{*}$ \\
\hline Interação & & $1,7^{\mathrm{ns}}$ & $2,5^{*}$ & $3,3 *$ & $2,1^{\mathrm{ns}}$ & $0,8^{\mathrm{ns}}$ & $0,7^{\mathrm{ns}}$ & $6,5^{*}$ \\
\hline Média geral & & 81,9 & 10.426 & 38,8 & 4.208 & 4,88 & 4,60 & 15,2 \\
\hline CV (\%) & & 24,7 & 26,9 & 13,4 & 14,3 & 13,9 & 11,1 & 15,1 \\
\hline
\end{tabular}

$\mathrm{MOR}$ = módulo de ruptura em flexão; $\mathrm{MOE}=$ módulo de elasticidade em flexão; $\mathrm{RC}=$ resistência à compressão paralela às fibras, $\mathrm{EC}=$ módulo de elasticidade em compressão paralela às fibras; DurA = dureza paralela às fibras; DurT = dureza perpendicular às fibras; $\mathrm{Cis}=$ resistência ao cisalhamento; $\mathrm{CV}=$ coeficiente de variação; * *ignificativo a $5 \%$ de probabilidade de erro. Médias seguidas da mesma letra, na coluna, não diferem entre si pelo teste de Tukey, ao nível de $5 \%$ de probabilidade de erro.

Tabela 3. Correlação de Pearson entre as propriedades físico-mecânicas da madeira de árvores de Hovenia dulcis presentes em remanescentes de floresta natural no município de Irati, PR.

Table 3. Pearson correlation between the physical-mechanical properties of Hovenia dulcis wood from natural forest remnants in the Municipality of Irati, PR.

\begin{tabular}{llllllllll}
\hline & TUe & MEa & MOR & MOE & RC & EC & DurA & DurT & Cis \\
\hline TUe & 1 & & & & & & & & \\
MEa & $0,320^{*}$ & 1 & & & & & & \\
MORF & $0,149^{\text {ns }}$ & $0,807^{*}$ & 1 & & & & & \\
MOEF & $-0,014^{\text {ns }}$ & $0,735^{*}$ & $0,892^{*}$ & 1 & & & & \\
RC & $-0,246^{\text {ns }}$ & $0,601^{*}$ & $0,496^{*}$ & $0,508^{*}$ & 1 & & & \\
EC & $-0,056^{\text {ns }}$ & $0,595^{*}$ & $0,674^{*}$ & $0,762^{*}$ & $0,804^{*}$ & 1 & & \\
DurA & $0,117^{\text {ns }}$ & $0,738^{*}$ & $0,686^{*}$ & $0,640^{*}$ & $0,488^{*}$ & $0,522^{*}$ & 1 & & \\
DurT & $-0,010^{\text {ns }}$ & $0,774^{*}$ & $0,640^{*}$ & $0,653^{*}$ & $0,647^{*}$ & $0,635^{*}$ & $0,941^{*}$ & 1 & \\
Cis & $-0,353^{*}$ & $0,370^{*}$ & $0,322^{*}$ & $0,382^{*}$ & $0,594^{*}$ & $0,481^{*}$ & $0,247^{\text {ns }}$ & $0,297^{\text {ns }}$ & 1 \\
\hline
\end{tabular}

$\mathrm{MEa}(\mathrm{M})=$ massa específica aparente; $\mathrm{MOR}$ = módulo de ruptura em flexão estática; $\mathrm{MOE}$ = módulo de elasticidade em flexão estática; $\mathrm{RC}=$ resistência à compressão paralela às fibras, $\mathrm{EC}=$ módulo de elasticidade em compressão paralela às fibras; DurA $=$ dureza paralela às fibras; DurT $=$ dureza perpendicular às fibras; $\mathrm{Cis}=$ resistência ao cisalhamento. ${ }^{*}$ Correlação significativa ao nível de $5 \%$ de probabilidade de erro; ns = Não significativo.

De acordo com a norma NBR 7190/97 (Associação Brasileira de Normas Técnicas, 1997), para madeiras de dicotiledôneas os valores médios de MEa, resistência à compressão, cisalhamento e módulo de elasticidade em compressão para a classe C20 são de $0,65 \mathrm{~g} \mathrm{~cm}^{-3} ; 20 \mathrm{MPa} ; 4 \mathrm{MPa}$ e $9.500 \mathrm{MPa}$, respectivamente. 

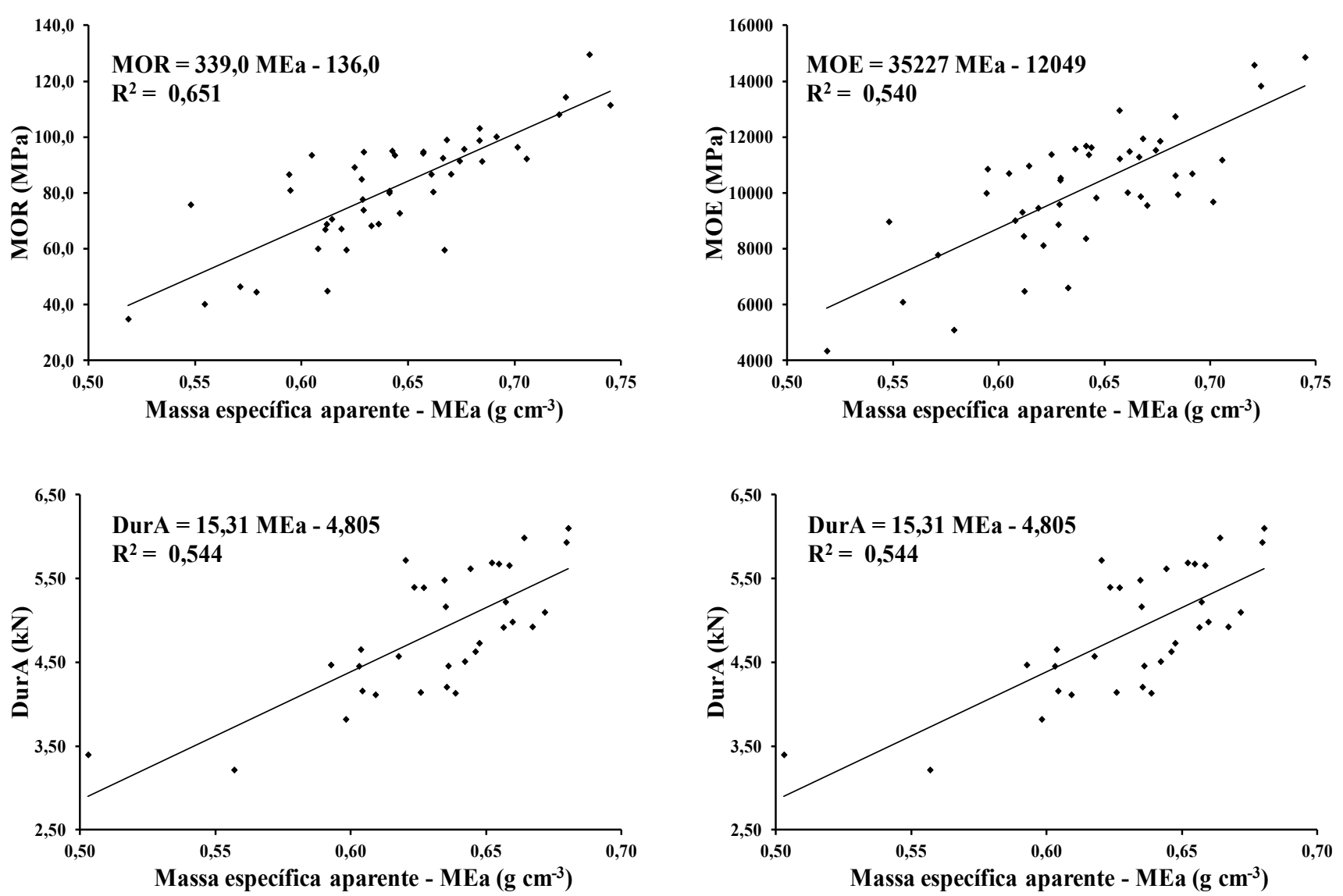

Figura 1. Propriedades mecânicas em função da massa específica aparente, com respectivas equações de regressão linear, da madeira de árvores de Hovenia dulcis provenientes de em remanescentes de floresta natural no município de Irati, PR. MOR = módulo de ruptura em flexão estática; $\mathrm{MOE}=$ módulo de elasticidade em flexão estática; DurA = dureza paralela às fibras; DurT $=$ dureza perpendicular às fibras.

Figure 1. Mechanical properties as a function of the apparent density, and their respective linear regression equations, of Hovenia dulcis wood from natural forest remnants in the Municipality of Irati, PR. MOR = modulus of rupture; MOE $=$ modulus of elasticity; DurA = hardness parallel to the fibers; DurT = hardness perpendicular to the fibers.

Considerando que os valores referenciais da norma são os valores característicos e corrigidos para $12 \%$ de TUe, verificou-se que a madeira de $H$. dulcis apresentou valores médios de propriedades mecânicas compatíveis ou maiores que a classificação C20, exceto para o módulo de elasticidade em compressão.

Sales (2004) estudou 42 espécies de madeiras brasileiras nativas e de reflorestamento, verificando que na classe $\mathrm{C} 20$ foram classificadas apenas quatro espécies (Cedrella odorata, Cedrella sp., Eucalyptus grandis e Erisma uncinatum), sendo as demais classificadas nas classes superiores. Dessa forma, comparando a classificação de $H$. dulcis com outras madeiras brasileiras, e por não atingir o requisito mínimo de rigidez (módulo elástico em compressão), verifica-se que sua madeira apresenta menor resistência mecânica.

\section{Conclusões}

Os valores médios das propriedades mecânicas estudadas permitem enquadrar a madeira de $H$. dulcis na classe C20 de classificação para madeiras de dicotiledôneas da Norma NBR 7190/97, exceto para módulo de elasticidade em compressão paralela. Dessa forma, devido à baixa rigidez e resistência moderada, sua madeira apresentou propriedades mecânicas que a tornam mais apropriada para uso não estrutural e para produção de móveis. 


\section{Referências}

Araújo, H. J. B. Relações funcionais entre propriedades físicas e mecânicas de madeiras tropicais brasileiras. Floresta, v. 37, n. 3, p. 399-416, 2007.

Associação Brasileira de Normas Técnicas. NBR 7190: projeto de estruturas de madeira. Rio de Janeiro, 1997.

Candaten, L. et al. Variação axial da massa específica básica de Hovenia dulcis. Enciclopédia Biosfera, v. 14 n. 26, p. 729-736, 2017. DOI: 10.18677/EnciBio_2017B67.

Carvalho, P. E. R. Ecologia, silvicultura e usos da uva-do-japão (Hovenia dulcis Thunberg). Colombo: EMBRAPA-CNPF, 1994. 24 p. (EMBRAPA-CNPF. Circular técnica, 23).

Carvalho, D. E. et al. Resistência natural de quatro espécies florestais submetidas a ensaio com fungos apodrecedores. Floresta e Ambiente, v. 22, n. 2, p. 271-276, 2015. DOI: 10.1590/21798087.105914.

Comisión Panamericana de Normas Técnicas. Norma Panamericana 460: maderas: método de determinación de la humedad. 1972a.

Comisión Panamericana de Normas Técnicas. Norma Panamericana 461: maderas: método de determinación del peso específico aparente. 1972b.

Comisión Panamericana de Normas Técnicas. Norma Panamericana 463: maderas: método de determinación del cizallamiento paralelo al grano. $1972 \mathrm{c}$.

Comisión Panamericana de Normas Técnicas. Norma Panamericana 464: maderas: método de determinación de la compresión axil o paralela al grano. $1972 \mathrm{~d}$.

Comisión Panamericana de Normas Técnicas. Norma Panamericana 465: maderas: método de determinación de la dureza. 1972e.

Comisión Panamericana de Normas Técnicas. Norma Panamericana 555: maderas: método de ensayo de flexión estática. 1973.

Del Menezzi, C. H. S. Estimativa das propriedades de flexão estática de seis espécies de madeiras amazônicas por meio da técnica nãodestrutiva de ondas de tensão. Acta Amazonica, v. 40, n. 2, p. 325-332, 2010.

Dias, F. M. \& Lahr, F. A. R. Estimativa de propriedades de resistência e rigidez da madeira através da densidade aparente. Scientia Forestalis, n. 5, p. 102-113, 2004.

Eleotério, J. R. at al. Crescimento em diâmetro, altura e volume de Hovenia dulcis na região Sul de Blumenau, SC. Floresta, v. 42, n. 4, p. 733-740, 2012. DOI: 10.5380/rf.v42i4.25054.

Eleotério, J. R. et al. Variação longitudinal da massa específica da madeira e da casca de Hovenia dulcis Thunberg. Ciência da Madeira, v. 6, n. 2, p. 122-128, 2015. DOI: 10.12953/2177-6830/ rcm.v6n2p122-128.

Forest Products Laboratory (United States). Wood handbook: wood as an engineering material. Madison, WI: U.S. Department of Agriculture, Forest Service, 2010. 508 p. (General technical report FPL-GTR-190).
Instituto Agronômico do Paraná. Cartas climáticas do Paraná. 2015. Disponível em <http://www.iapar.br/modules/conteudo/ conteudo.php?conteudo=597>. Acesso em: 1 set. 2015.

Mendes, L. M. \& Arce, J. E. Análise comparativa das equações utilizadas para estimar a umidade de equilíbrio da madeira. Cerne, v. 9, n. 2, p. 141-152, 2003.

Müller, B. V. et al. Avaliação das principais propriedades físicas e mecânicas da madeira de Eucalyptus benthamii Maiden et Cambage. Floresta e Ambiente, v. 21, n. 4, p. 535-542, 2014. DOI: 10.1590/2179-8087.050413.

Napoli, L. M. et al. Propriedades físicas da madeira e de painéis aglomerados produzidos com misturas de espécies florestais. Floresta, v. 43, n. 3, p. 475-484, 2013.

Rigatto, P. A. et al. Características físicas, químicas e anatômicas da madeira de Hovenia dulcis. Colombo: EMBRAPA-CNPF, 2001. 5 p. (EMBRAPA-CNPF. Comunicado técnico, 66).

Sales, A. Sistema de classes de resistência para dicotiledôneas: revisão da NBR 7190/97. Madeira: arquitetura e engenharia, n. 13, artigo 9, p. 1-11, 2004.

Schumacher, M. V. et al. Biomassa e nutrientes em um povoamento de Hovenia dulcis Thunb., plantado na Fepagro florestas, Santa Maria, RS. Ciência Florestal, v. 18, n. 1, p. 27-37, 2008. DOI: 10.5902/19805098519.

Susin, F. et al. Taxa de secagem e qualidade da madeira serrada de Hovenia dulcis submetida a dois métodos de secagem. Floresta e Ambiente, v. 2, n. 2, p. 243-250, 2014. DOI: 10.4322/ floram.2014.016.

Talgatti, M. et al. Massa específica aparente e suas implicações na flexão dinâmica da madeira de Hovenia dulcis Thunb. Scientia Agraria Paranaensis, v. 16, n. 1, p. 21-26, 2017. DOI: 10.18188/1983-1471/sap.v16n1p21-26.

Tenorio, C. et al. Evaluation of wood properties from six native species of forest plantations in Costa Rica. Bosque, v. 37, n. 1, p. 71-84, 2016. DOI: 10.4067/S0717-92002016000100008.

Tomazeli, A. J. et al. Durabilidade natural de quatro espécies florestais em campo de apodrecimento. Tecno-Lógica, v. 20, n. 1, p. 20-25, 2015. DOI: 10.17058/tecnolog.v20i1. 6473.

Trevisan, R. et al. Axial variation of basic density of Araucaria angustifolia wood in different diameter classes. Ciência Rural, v. 46, n. 11, p. 1969-1972, 2016. DOI: 10.1590/0103-8478cr20141312.

Vidaurre, G. et al. Lenho juvenil e adulto e as propriedades da madeira. Floresta e Ambiente, v. 18, n. 4, p. 469-480, 2011. DOI: 10.4322/floram.2011.066.

Vivian, M. A. et al. Efeito de pré-tratamentos na taxa de secagem da madeira de Hovenia Dulcis Thunb. Ciência da Madeira, v. 1, n. 2, p. 32-43, 2010. 